\title{
(6) OPEN ACCESS \\ Native valve disease in patients with non-valvular atrial fibrillation on warfarin or rivaroxaban
}

\author{
Günter Breithardt, ${ }_{1}^{1}$ Helmut Baumgartner, ${ }^{2}$ Scott D Berkowitz, ${ }^{3}$ Anne S Hellkamp, ${ }^{4}$ \\ Jonathan P Piccini, ${ }^{4}$ Yuliya Lokhnygina, ${ }^{4}$ Jonathan L Halperin, ${ }^{5}$ Daniel E Singer ${ }^{6}{ }^{6}$ \\ Graeme J Hankey, ${ }^{7}$ Werner Hacke, ${ }^{8}$ Richard C Becker, ${ }^{9}$ Christopher C Nessel, ${ }^{10}$ \\ Kenneth W Mahaffey, ${ }^{11}$ Robert M Califf, ${ }^{12}$ Keith A A Fox ${ }_{1}^{13}$ Manesh R Patel, ${ }^{4}$ \\ for the ROCKET AF Steering Committee \& Investigators
}

\begin{abstract}
- Additional material is published online only. To view please visit the journal online (http://dx.doi.org/10.1136/ heartjnl-2015-308120).
\end{abstract}

For numbered affiliations see end of article.

\section{Correspondence to} Professor Günter Breithardt, Division of Electrophysiology, Department of Cardiovascular Medicine, University Hospital Münster, Münster, Germany; g.breithardt@uni-muenster.de

These data were partly presented at the Annual Congress of the European Society of Cardiology 2014 in Barcelona.

Received 21 May 2015 Revised 20 January 2016 Accepted 26 January 2016 Published Online First 17 February 2016

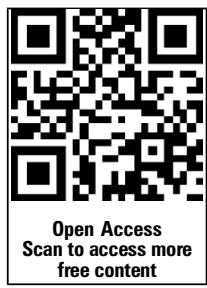

\section{(1) cossmalk}

To cite: Breithardt G, Baumgartner $\mathrm{H}$, Berkowitz SD, et al. Heart 2016;102:1036-1043.

\section{ABSTRACT \\ Objective To compare the characteristics and} outcomes of patients with atrial fibrillation (AF) and aortic stenosis (AS) with patients with AF with mitral regurgitation (MR) or aortic regurgitation $(A R)$ and patients without significant valve disease (no SVD). Methods Using Rivaroxaban Once-Daily, Oral, Direct Factor Xa Inhibition Compared With Vitamin K Antagonism for Prevention of Stroke and Embolism Trial in Atrial Fibrillation (ROCKET AF) data, we analysed efficacy and safety outcomes, adjusting hazard ratios (HRs) for potential confounders using Cox regression analysis.

Results Among 14119 intention-to-treat ROCKET AF trial patients, a trial that excluded patients with mitral stenosis or artificial valve prosthesis, 214 had AS with or without other valve abnormalities, 1726 had MR or AR and 12179 had no SVD. After adjusting for prognostic factors, the composite of stroke, systemic embolism or vascular death increased approximately twofold in patients with AS (AS 10.84, MR or AR 4.54 and no SVD 4.31 events per 100 patient-years, $p=0.0001)$. All-cause death also significantly increased (AS 11.22, MR or AR 4.90 and no SVD 4.39 events per 100 patient-years, $p=0.0003$ ). Major bleeding occurred more frequently in AS (adjusted HR 1.61, confidence intervals (CI) 1.03 to 2.49, $\mathrm{p}<0.05$ ) and MR or AR (HR 1.30, 1.07 to 1.57, $\mathrm{p}<0.01$ ) than in no SVD, but there was no difference between $A S$ and MR or AR (HR $1.24,0.78$ to 1.97$)$. The relative efficacy of rivaroxaban versus warfarin was consistent among patients with and without valvular disease. Rivaroxaban was associated with higher rates of major bleeding than warfarin in patients with MR or AR (HR 1.63, 1.15 to 2.31).

Conclusions We found that patients with AF and AS on oral anticoagulants may have distinctly different efficacy and safety outcomes than patients with MR or AR or no SVD.

Trial registration number NCT00403767;

Post-results.

\section{INTRODUCTION}

The Rivaroxaban Once-Daily, Oral, Direct Factor $\mathrm{Xa}$ Inhibition Compared With Vitamin K Antagonism for Prevention of Stroke and Embolism Trial in Atrial Fibrillation (ROCKET AF) study was a large randomised trial that compared warfarin and rivaroxaban, a novel factor $\mathrm{Xa}$ inhibitor, in patients with non-valvular AF, defined as the absence of mitral stenosis or artificial valve prosthesis. ${ }^{12}$ These criteria allowed for the inclusion of patients with other valve disease types, representing a heterogeneous group with mitral regurgitation (MR), aortic regurgitation (AR) or aortic stenosis (AS), alone or in combination. ${ }^{3}$ The clinical characteristics and outcomes of patients with AF with valve disease, compared with those without, were recently reported. ${ }^{3}$

In our retrospective study, we compared the characteristics and outcomes of patients with ROCKET AF without valve disease ('true' nonvalvular AF) with two subgroups with valve disease. We hypothesised that patients with AS on oral anticoagulation might have different outcomes than patients with MR or AR.

\section{METHODS}

We used ROCKET AF data to retrospectively perform our analysis. ${ }^{1} 2$ The rationale and design of ROCKET AF have been previously published (ClinicalTrials.gov \#NCT00403767). ${ }^{1}$ Our study differed from the previously published analysis ${ }^{3}$ since we compared two subgroups of patients with valve disease with patients with non-valvular AF. If the recruiting physician considered a lesion to be significant, then the patient was included in the group with significant valve disease (SVD). Patients were excluded if they met clinical criteria for imminent surgical or percutaneous valvular intervention, therefore excluding those with advanced/high-grade valvular disease. Patients with SVD were subdivided into those with AS (if at least one valve location/ abnormality was AS) and those with MR or AR (table 1), which included patients in whom at least one valve location/abnormality was MR or AR (but none was AS). For the purposes of this study, we analysed patients with: (1) no SVD and subgroups with SVD, (2) AS and (3) MR or AR.

Among 14171 intention-to-treat patients, 12179 had no SVD, whereas 1992 (14.1\%) had investigator-classified SVD. In 52 patients with SVD, the valve location was unknown; consequently, they were omitted from the current efficacy analyses, leaving 1940 intention-to-treat patients with SVD (AS, $n=214$; MR or AR, $n=1726$ ). The safety population consisted of 14236 , including 1945 patients with SVD with known location, 12237 without SVD and 54 patients were omitted for SVD with unknown location.

The primary efficacy endpoint in ROCKET AF was the composite of all stroke (both ischaemic and 
Table 1 Location of valvular disease in those 1940 randomised ITT patients in whom information on the location of diseased valves was available

\begin{tabular}{lr}
\hline Type of valve disease & N \\
\hline Aortic stenosis ( $\mathrm{n}=214)$ & \\
Isolated aortic stenosis & 87 \\
Plus mitral regurgitation & 59 \\
Plus aortic regurgitation & 18 \\
Plus mitral and aortic regurgitation & 50 \\
Mitral or aortic regurgitation ( $\mathrm{n}=1726)$ & \\
Isolated mitral regurgitation & 1309 \\
Mitral and aortic regurgitation & 329 \\
Isolated aortic regurgitation & 88 \\
\hline ITT, intention to treat. &
\end{tabular}

haemorrhagic) and systemic embolism. ${ }^{1}{ }^{2}$ Secondary efficacy endpoints included stroke; all-cause death; the composite of stroke, systemic embolism or vascular death; and the composite of stroke, systemic embolism, vascular death or myocardial infarction. The primary safety endpoint was major or non-major clinically relevant bleeding (NMCR); secondary safety endpoints were major bleeding, gastrointestinal bleeding and intracranial haemorrhage. All clinical endpoints were adjudicated by a committee blinded to the treatment assignment.

\section{Statistical analysis}

Two populations were used: (1) intention to treat: patients who were randomised, for baseline summaries and analysis of efficacy endpoints and (2) on-treatment (or safety): intention-to-treat patients who received at least one study drug dose, for safety endpoint analysis. The methodology of the present analyses has been reported $^{3}$ (see online supplementary material).

Clinical characteristics are presented using medians (with 25 th and 75 th percentiles) for continuous variables and percent (frequency) for categorical variables. A single overall test (Kruskal-Wallis for continuous variables and Pearson $\chi^{2}$ for categorical) was performed to determine the differences among the three subgroups.

Event rates (per 100 patient-years and total) were generated for all endpoints. Cox regression analysis was used to adjust comparisons for potential confounders. ${ }^{3}$ Hazard ratios (HRs; 95\% confidence intervals (CIs)) and Wald $\chi^{2}$ tests were generated for all three pairwise comparisons: (1) AS versus MR or AR; (2) AS versus no SVD and (3) MR or AR versus no SVD.

The efficacy analyses used the intention-to-treat population and the time period to site notification. The safety endpoint analyses used the safety population and the on-treatment period (ie, time to last exposure plus 2 days). The same covariates were used as reported $\left({ }^{3}\right.$; see online supplementary material). For treatment comparisons among subgroups, event rates were summarised by treatment among AS, MR or AR, and no SVD. After adding interaction terms AS*rivaroxaban and other-SVD*rivaroxaban, a single overall test was performed in each model to determine treatment effect differences among the three groups; if significant, then rivaroxaban versus warfarin HRs (95\% CI) were generated for all three subgroups. For each model, the proportional hazards assumption was tested and found to have been met.

All statistical analyses were performed by the Duke Clinical Research Institute (Durham, North Carolina, USA) using SAS software (V.9.2, SAS Institute, Cary, North Carolina, USA).

\section{RESULTS}

\section{Clinical characteristics of patients}

Tables 1 and 2 show the location of valve disease and clinical characteristics of 1940 intention-to-treat patients. Compared with others, patients with AS were older, slightly heavier, less often had persistent AF, more new onset AF, more peripheral artery disease, prior myocardial infarction, slightly higher $\mathrm{CHADS}_{2}$ (C, Congestive heart failure; H, Hypertension; A, Age $\geq 75$ years; D, Diabetes mellitus; $S_{2}$, prior Stroke or transient ischaemic attack (TIA) or thromboembolism) score and lower creatinine clearances.

\section{Efficacy and safety endpoints}

Event rates for efficacy and safety outcomes are presented in figures 1 and 2, table 3 and in online supplementary figures 1 and 2. Stroke or systemic embolism occurred about twice as often in the AS group (4.21) than in the MR or AR (2.01) or the no SVD groups (2.09 events/100 patient-years; $\mathrm{p}<0.05$ each), whereas there was no difference between patients with MR or AR and patients with no SVD. The composite of stroke, systemic embolism or vascular death was increased approximately twofold in patients with AS (AS 10.84) compared with patients with MR or AR (4.54) and patients with no SVD (4.31 events per 100 patient-years, $\mathrm{p}<0.01$ each), whereas again, MR or AR and no SVD did not differ. All-cause death (AS 11.22, MR or AR 4.90 and no SVD 4.39 events per 100 patient-years) and the combined outcome of stroke, systemic embolism and vascular death (with or without myocardial infarction) occurred also twice as often in AS than in the other two groups $(p<0.01$ for each comparison), whereas MR or AR did not differ from no SVD (table 3B).

Early study drug discontinuation rates were different in the three groups (AS 58\%, MR or AR 40\% and no SVD 34\%). However, patterns of stroke rates in the per-protocol on-treatment population were not markedly different from those in intention-to-treat patients (table $3 \mathrm{~A}$ ).

Major bleeding (figure 2 and table 3B, see online supplementary figure S2) occurred more frequently in AS (7.61) than in no SVD (3.27 events/100 patient-years; adjusted HR 1.61, CI 1.03 to $2.49, \mathrm{p}<0.05)$. In patients with $\mathrm{MR}$ or $\mathrm{AR}$, the risk of major bleeding was also significantly higher (4.86 events/100 patientyears) than in no SVD (adjusted HR 1.30, CI 1.07 to 1.57 , $\mathrm{p}<0.01$ ), whereas the difference between AS and MR or AR did not reach significance (adjusted HR 1.24, CI 0.78 to 1.97). Major clinically relevant and NMCR bleeding occurred more often in AS (24.36), less often in MR or AR (17.66) and was lowest in no SVD (14.16 events/100 patient-years). However, only the difference between MR or AR and no SVD reached significance $(p<0.05)$. Gastrointestinal bleeding rates were numerically higher in AS and MR or AR, but statistically different only for MR or AR versus no SVD $(\mathrm{p}<0.05)$. Intracranial haemorrhage occurred rarely and did not differ among groups (table $3 \mathrm{~B}$ ).

\section{Treatment comparisons for efficacy and safety endpoints}

There were no significant interactions among patients randomised to rivaroxaban and warfarin, indicating a consistent risk relationship between rivaroxaban and warfarin across the three subgroups for all efficacy endpoints (see online supplementary table S1). For the major or NMCR bleeding and major bleeding outcomes, the interaction tests were significant (figure 3, see online supplementary table S1). In patients without SVD, rivaroxaban and warfarin had a neutral relationship (HRs very close to 1), while patients with MR or AR had an elevated risk of 
Table 2 Baseline clinical characteristics for all ITT patients with known location of valve disease grouped by the three patient subgroups

\begin{tabular}{|c|c|c|c|c|}
\hline Variable & $A S^{*}(n=214)$ & MR or $A^{*}(n=1726)$ & No SVD* $(n=12179)$ & $\mathrm{p}$ Valuet \\
\hline Age & $78(73,82)$ & $74(67,79)$ & $72(65,78)$ & $<0.0001$ \\
\hline Female & $37 \%(79)$ & $40 \%(682)$ & $40 \%(4820)$ & 0.73 \\
\hline Atrial fibrillation & & & & 0.0082 \\
\hline Persistent & $79 \%(170)$ & $83 \%(1435)$ & $81 \%(9832)$ & \\
\hline Paroxysmal & $17 \%(37)$ & $16 \%(277)$ & $18 \%(2173)$ & \\
\hline New onset & $3 \%(7)$ & $1 \%(14)$ & $1 \%(174)$ & \\
\hline $\mathrm{CHADS}_{2}$ score (mean (SD)) & $3.6(0.9)$ & $3.5(1.0)$ & $3.5(0.9)$ & 0.053 \\
\hline \multicolumn{5}{|l|}{$\mathrm{CHADS}_{2}$ score } \\
\hline 2 & $8 \%(18)$ & $14 \%(247)$ & $13 \%(1585)$ & \\
\hline 3 & $42 \%(89)$ & $43 \%(743)$ & $44 \%(5311)$ & \\
\hline 4 & $33 \%(70)$ & $27 \%(467)$ & $29 \%(3521)$ & \\
\hline 5 & $14 \%(30)$ & $13 \%(230)$ & $13 \%(1532)$ & \\
\hline 6 & $3 \%(7)$ & $2 \%(39)$ & $2 \%(230)$ & \\
\hline HAS-BLED score (mean (SD)) & $3.0(0.9)$ & $2.8(1.0)$ & $2.8(0.9)$ & 0.0005 \\
\hline \multicolumn{5}{|l|}{ Presenting characteristics } \\
\hline $\mathrm{BMI}, \mathrm{kg} / \mathrm{m}^{2}$ & $29(26,32)$ & $28(25,31)$ & $28(25,32)$ & $<0.0001$ \\
\hline Systolic BP, mm Hg & $130(120,142)$ & $130(120,140)$ & $130(120,140)$ & $<0.0001$ \\
\hline Diastolic BP, mm Hg & $78(70,85)$ & $80(70,85)$ & $80(70,86)$ & $<0.0001$ \\
\hline Creatinine clearance, $\neq \mathrm{ml} / \mathrm{min}$ & $61(44,78)$ & $63(49,80)$ & $68(53,88)$ & $<0.0001$ \\
\hline \multicolumn{5}{|l|}{ Baseline comorbidities } \\
\hline Prior stroke, TIA or non-CNS embolism & $46 \%(99)$ & $48 \%(829)$ & $56 \%(6806)$ & $<0.0001$ \\
\hline PAD & $15 \%(32)$ & $7 \%(124)$ & $6 \%(672)$ & $<0.0001$ \\
\hline Hypertension & $92 \%(197)$ & $89 \%$ (1542) & 91\% (11 049) & 0.14 \\
\hline Diabetes & $43 \%(92)$ & $40 \%(690)$ & $40 \%(4849)$ & 0.64 \\
\hline Prior Ml & $30 \%(64)$ & $23 \%(404)$ & $16 \%(1964)$ & $<0.0001$ \\
\hline Congestive HF & $65 \%(139)$ & $71 \%(1228)$ & $61 \%(7449)$ & $<0.0001$ \\
\hline COPD & $19 \%(40)$ & $14 \%(243)$ & $10 \%(1194)$ & $<0.0001$ \\
\hline \multicolumn{5}{|l|}{ Medications } \\
\hline Prior vitamin $\mathrm{K}$ antagonist use & $74 \%(158)$ & $72 \%(1251)$ & $61 \%(7409)$ & $<0.0001$ \\
\hline Prior chronic ASA use & $37 \%(80)$ & $34 \%(589)$ & $37 \%(4494)$ & 0.079 \\
\hline ACE-inhibitor/ARB at baseline & $78 \%(167)$ & $76 \%(1306)$ & $74 \%(9029)$ & 0.18 \\
\hline$\beta$ blocker at baseline & $65 \%(140)$ & $71 \%(1225)$ & $64 \%(7789)$ & $<0.0001$ \\
\hline Digitalis at baseline & $41 \%(88)$ & $44 \%(754)$ & $38 \%(4589)$ & $<0.0001$ \\
\hline Diuretic at baseline & $75 \%(160)$ & $70 \%(1212)$ & $58 \%(7031)$ & $<0.0001$ \\
\hline Randomised to rivaroxaban & $49 \%(105)$ & $48 \%(834)$ & $50 \%(6119)$ & 0.32 \\
\hline
\end{tabular}

All other abbreviations can be found in table 1 .

${ }^{*}$ Continuous variables are shown as median (25th, 75th percentile) except where noted, and categorical variables as \% (n).

tp Values are for any differences across groups.

$\neq$ Cockroft-Gault.

A, Age $\geq 75$ years; ARB, angiotensin receptor blocker; AS, aortic stenosis; ASA, acetylsalicylic acid, aspirin; HAS-BLED, hypertension, abnormal renal/liver function, stroke, bleeding history or predisposition, labile international normalized ratio, elderly ( $>65$ years), drugs/alcohol concomitantly; BMI, body mass index; BP, blood pressure; CHADS 2 , C, congestive heart failure; CNS, central nervous system; COPD, chronic obstructive pulmonary disease; $D$, diabetes mellitus; $H$, hypertension; $H F$, heart failure; MI, myocardial infarction; MR or AR, mitral or aortic regurgitation; PAD, peripheral artery disease; $S_{2}$, prior Stroke or TIA or thromboembolism; SD, standard deviation; SVD, significant valve disease; TIA, transient ischaemic attack; $R$, rivaroxaban; $W$, warfarin.

either of these two bleeding categories with rivaroxaban compared with warfarin (HR 1.32 and 1.63, respectively). Patients with AS also had HRs of 1.18 and 1.73 , but the CIs were wide, reaching 1.0. There was no significant interaction for gastrointestinal bleeding, and there were too few intracranial haemorrhages to draw any conclusions (see online supplementary table S1).

\section{DISCUSSION}

In ROCKET AF, $14.1 \%$ of patients had SVD that did not meet mitral stenosis and artificial valve prostheses exclusion criteria ${ }^{3}$; these patients were older and had more comorbidities than those without SVD, similar to preliminary data from the Randomised Evaluation of Long Term Anticoagulant Therapy (RE-LY) and Apixaban for Reduction in Stroke and Other Thromboembolic Events in Atrial Fibrillation (ARISTOTLE) trials. ${ }^{4-6}$ In ROCKET AF, the rates of stroke or systemic embolism were comparable among patients with and without SVD, whereas the rates of major or NCMR or major bleeding alone were higher in patients with $\mathrm{SVD}^{3}{ }^{3}$ similar to findings from ARISTOTLE $^{45}$ and RE-LY. ${ }^{4}$ In patients with SVD allocated to rivaroxaban, the rates of major and NMCR or major bleeding alone were higher than in warfarin patients compared with no-SVD patients. However, there was no difference between both treatment arms after controlling for risk factors and potential confounders. For intracranial haemorrhage, there was no interaction between patients with and without SVD where the overall rate was lower among those randomised to rivaroxaban. ${ }^{3}$ Our patients with $\mathrm{SVD}^{3}$ were older (median 75 vs 71 years), had higher $\mathrm{CHADS}_{2}$ scores (mean 3.5 vs 2.2) and had more frequently prior stroke, systemic embolism or TIA $(48.2 \%$ vs $18.8 \%$ ) than patients with valvular heart disease in ARISTOTLE. 5

Preliminary data for our retrospective exploratory analysis were recently presented. ${ }^{7}$ Here, we present a detailed analysis of 
Figure 1 Efficacy endpoints by SVD subtype and for patients with no SVD based on ITT patients. Efficacy endpoints (events per 100 patient-years, unadjusted) by SVD subtype and for patients with no SVD based on ITT patients. $p$ Values for any difference among groups are based on Cox proportional hazards models. Patients in both treatment arms are combined. For results of detailed statistical analyses, see table 3 . ITT, intention to treat; MI, myocardial infarction; pt-years, patient-years; SE, systemic embolism; SVD, significant valve disease; MR or AR, mitral or aortic regurgitation; vasc. death, vascular death.

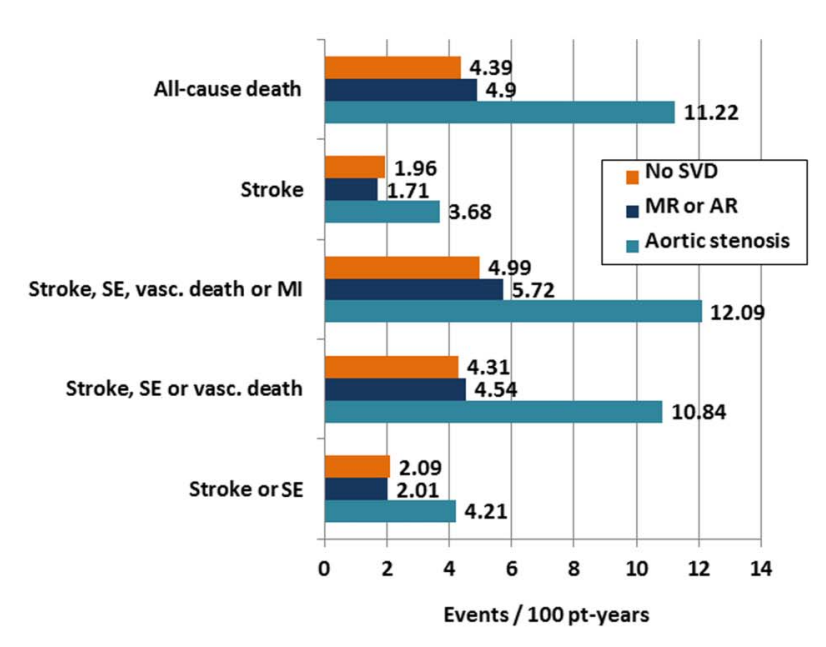

P-value for any difference among groups 0.0003 0.090 0.0002 $<0.0001$ 0.061

ITT patients

Cox proportional hazards models valve disease subgroups in patients with non-valvular AF treated with oral anticoagulation.

\section{Clinical characteristics}

Due to protocol inclusion and exclusion criteria, ${ }^{12}$ patients with AS suffered moderate-to-intermediate aortic lesions since severe lesions requiring imminent intervention were excluded. The majority of patients in the AS group (59\%) also had MR or AR (table 1). No reasonable comparison can be made between our patients (who constitute a special group, all with $\mathrm{AF}$ requiring anticoagulation for the prevention of stroke or systemic embolism) with other reports. ${ }^{8-12}$ These reports either included patients with aortic valve sclerosis, ${ }^{8}$ or AS without any anticoagulation, ${ }^{9}$ or patients with MR selected on the basis of left ventricular dysfunction who had a low prevalence of AF of $8-11 \%{ }^{10}$ or $8.8-16.3 \%{ }^{11}$ without any information on oral anticoagulation or no information on the prevalence of AF or oral anticoagulation. ${ }^{12}$

\section{Efficacy outcomes}

Our analysis suggests that secondary endpoints, including allcause death, were significantly higher among AS patients, despite AS being of moderate or intermediate severity. Also, stroke and systemic embolism (the primary outcome parameter) occurred about twice as often in patients with AS compared with those without (table 3).

Clinical outcomes like stroke, systemic embolism or death among patients with valvular disease subtypes treated with oral anticoagulation have not been well established. The association between AS and stroke is controversial. ${ }^{13-16}$ The need for oral anticoagulation in patients with AS is generally not mentioned in valvular heart disease guidelines, ${ }^{14}{ }^{17}$ except when AF is present.

The rate of stroke in the AS group with AF on oral anticoagulation was high (3.68/100 patient-years) in comparison with the Simvastatin and Ezetimibe in Aortic Stenosis (SEAS) trial in which patients in the early phase of disease were selected for drug intervention (0.56/100 patient-years). ${ }^{9}$ SEAS only included patients not on oral anticoagulation; only $6.4 \%$ had AF. SEAS patients had mild-to-moderate AS (presumably similar in severity to our patients), but a much lower stroke risk, which correlated to a low $\mathrm{CHA}_{2} \mathrm{DS}_{2}$-VASc $(\mathrm{C}$, congestive heart failure; $\mathrm{H}$, hypertension; A, age $\geq 75$ years; $D$, diabetes mellitus; $S_{2}$, prior Stroke or TIA or thromboembolism; V, vascular disease; A, age 65-74 years; SC, sex category (female)) score of $1.8 \pm 1.2 .^{9}$ In our study, patients were markedly older (78 median vs 67.3 \pm 9.7 years), and all had $\mathrm{AF}$ and a 3.6 $\mathrm{CHADS}_{2}$ score (median).
Figure 2 Safety endpoints by SVD subtype and for patients with no SVD. Safety endpoints (events per 100 patient-years, unadjusted) by SVD subtype and for patients with no SVD. $p$ Values for any difference among groups are based on Cox proportional hazards models. Patients in both treatment arms are combined. For results of detailed statistical analyses, see table 3. NMCR, non-major clinically relevant; pt-years, patient years; SVD, significant valve disease; MR or AR, mitral or aortic regurgitation.

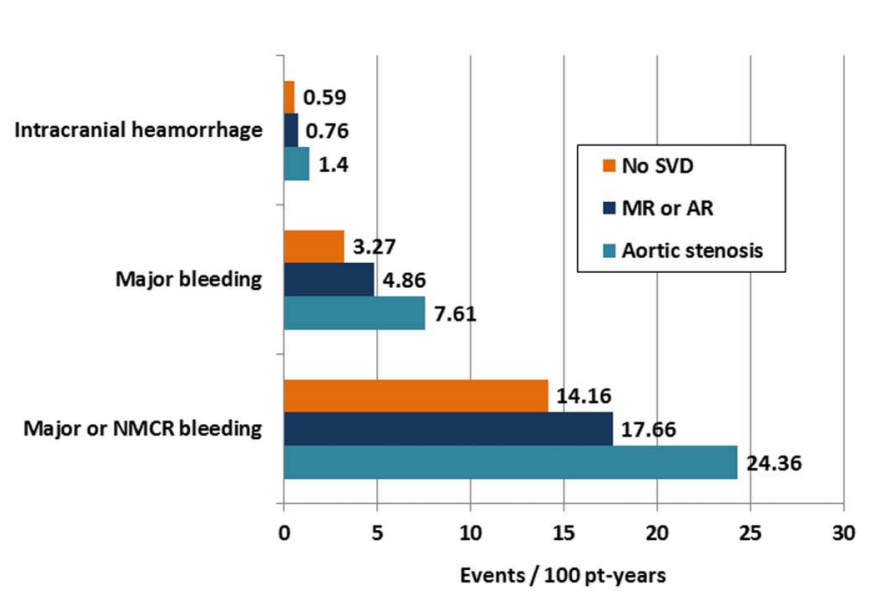

p-value for any

difference among groups

0.27

0.0049

0.030 
Table 3 Efficacy (ITT population) and safety endpoints (on-treatment population) by SVD subtype and for patients with no SVD

\begin{tabular}{|c|c|c|c|}
\hline \multicolumn{4}{|l|}{ (A) Event rates } \\
\hline Outcomes & $\begin{array}{l}\text { AS events } / 100 \text { pt-years } \\
\text { (CI) (total events) }\end{array}$ & $\begin{array}{l}\text { MR or AR events/100 pt-years } \\
\text { (CI) (total events) }\end{array}$ & $\begin{array}{l}\text { No SVD events } / 100 \text { pt-years } \\
\text { (CI) (total events) }\end{array}$ \\
\hline \multicolumn{4}{|l|}{ Efficacy outcomes } \\
\hline Stroke or SE & 4.21 (1.89 to 6.53$)(17)$ & 2.01 (1.50 to 2.52$)(69)$ & 2.09 (1.89 to 2.29$)(487)$ \\
\hline Stroke, SE, or vascular death & $10.84(7.23$ to 14.45$)(41)$ & 4.54 (3.78 to 5.30$)(152)$ & 4.31 (4.03 to 4.59$)(982)$ \\
\hline Stroke, SE, vascular death, or MI & 12.09 (8.25 to 15.93$)(45)$ & 5.72 (4.86 to 6.58$)(189)$ & 4.99 (4.68 to 5.30$)(1128)$ \\
\hline Stroke & $3.68(1.52$ to 5.84$)(15)$ & 1.71 (1.24 to 2.18$)(59)$ & 1.96 (1.77 to 2.15$)(458)$ \\
\hline Early study drug discontinuation (\%)* & $58.4(51.5$ to 65.1$)(125)$ & 39.6 (37.3 to 41.9$)(685)$ & 34.0 (33.2 to 34.8$)(4161)$ \\
\hline Stroke per-protocol on-treatment ${ }^{*}$ & 4.30 (1.81 to 6.79$)(12)$ & 1.50 (1.04 to 1.96$)(39)$ & $1.90(1.70$ to 2.10$)(354)$ \\
\hline All-cause death & $11.22(7.65$ to 14.79$)(43)$ & $4.90(4.12$ to 5.68$)(164)$ & 4.39 (4.11 to 4.67$)(1002)$ \\
\hline \multicolumn{4}{|l|}{ Safety outcomes } \\
\hline Major or NMCR bleeding & 24.36 (18.14 to 30.58) (59) & 17.66 (15.98 to 19.34$)(422)$ & 14.16 (13.60 to 14.72$)(2431)$ \\
\hline Major bleeding & $7.61(4.35$ to 10.87$)(21)$ & $4.86(4.03$ to 5.69$)(131)$ & 3.27 (3.01 to 3.53$)(625)$ \\
\hline Gastrointestinal bleeding & 2.90 (0.89 to 4.91$)(8)$ & 2.12 (1.57 to 2.67$)(57)$ & $1.32(1.16$ to 1.48$)(252)$ \\
\hline Intracranial haemorrhage & 1.40 (0.03 to 2.77$)(4)$ & $0.76(0.43$ to 1.09$)(21)$ & $0.59(0.48$ to 0.70$)(114)$ \\
\hline
\end{tabular}

(B) Group comparisons

\begin{tabular}{|c|c|c|c|}
\hline Outcomes & $\begin{array}{l}\text { AS vs No SVD } \\
\text { HR (CI) }\end{array}$ & $\begin{array}{l}\text { AS vs MR or AR } \\
\text { HR (CI) }\end{array}$ & $\begin{array}{l}\text { MR or AR vs no SVD } \\
\text { HR (CI) }\end{array}$ \\
\hline \multicolumn{4}{|l|}{ Efficacy outcomes } \\
\hline Stroke or SE & $1.82(1.10 \text { to } 3.01)^{*}$ & $1.87(1.08 \text { to } 3.23)^{*}$ & $0.97(0.75$ to 1.26$)$ \\
\hline Stroke, SE or vascular death & $2.03(1.47 \text { to } 2.79)^{* *}$ & $2.12(1.49 \text { to } 3.01)^{* *}$ & $0.96(0.80$ to 1.14$)$ \\
\hline Stroke, SE, vascular death or MI & $1.91(1.41 \text { to } 2.59)^{* *}$ & $1.84(1.32 \text { to } 2.56)^{* *}$ & $1.04(0.88$ to 1.21$)$ \\
\hline Stroke & $1.70(1.00 \text { to } 2.92)^{*}$ & $1.93(1.07 \text { to } 3.46)^{*}$ & $0.88(0.67$ to 1.16$)$ \\
\hline All-cause death & $1.88(1.38 \text { to } 2.56)^{* *}$ & $1.94(1.38 \text { to } 2.72)^{* *}$ & $0.97(0.82$ to 1.15$)$ \\
\hline \multicolumn{4}{|l|}{ Safety outcomes } \\
\hline Major or NMCR bleeding & 1.28 (0.98 to 1.66$)$ & $1.14(0.87$ to 1.51$)$ & $1.12(1.00 \text { to } 1.24)^{*}$ \\
\hline Major bleeding & $1.61(1.03 \text { to } 2.49)^{*}$ & 1.24 (0.78 to 1.97$)$ & $1.30(1.07 \text { to } 1.57)^{* *}$ \\
\hline Gastrointestinal bleeding & 1.39 (0.68 to 2.83$)$ & 1.04 (0.49 to 2.18$)$ & $1.34(1.00 \text { to } 1.80)^{*}$ \\
\hline Intracranial haemorrhage & $2.04(0.75$ to 5.60$)$ & $1.63(0.55$ to 4.77$)$ & $1.26(0.79$ to 2.01$)$ \\
\hline
\end{tabular}

Therefore, the higher rate of stroke and systemic embolism in our cohort compared with $\mathrm{SEAS}^{9}$ may be explained by the presence of AF, older age and other risk factors impacting the stroke risk score.

In a study from Olmsted County, ${ }^{18} 22 \%$ of patients with AS experienced a cerebrovascular event during 7-year follow-up (adjusted yearly risk 3.1\%), which seems comparable to ROCKET AF. Yet, the Olmsted County analysis excluded systemic embolism and included patients with severe AS (39\% of cases); 48\% received oral anticoagulation. Following adjustment for age and AF, severe AS (compared with moderate) was associated with an increased risk of cerebrovascular events (risk ratio $5.35,95 \%$ CI 1.4 to 8.6$).^{18}$

The death rate in patients with AS was about twice as high as those with MR or AR and no SVD $(p<0.01$ each). In the Olmsted County study, ${ }^{18}$ patients with AS had increased mortality ( $57 \%$ at 5 years; $71 \%$ at 7 years), which is not too different from our death rate (11.22/100 patient-years) when compared with their 5-year and 7-year rates. Nevertheless, as previously noted, the Olmsted County study also included patients with severe AS, who have a 50\% 1-year mortality rate.

MR was the dominant diagnosis in the MR or AR group. The risk of stroke or systemic embolism in patients with MR or AR was comparable with no SVD. Some reports suggest that patients with AF with MR have lower rates of left atrial thrombi intra-operatively ${ }^{19}{ }^{20}$ or on echocardiography ${ }^{21}$ than those without MR. Some suggest a reduced propensity to thromboembolism in patients with $\mathrm{MR}$ and $\mathrm{AF}^{22-24}$ although other studies failed to show a similar beneficial effect of $M R .{ }^{25}$ In patients with non-rheumatic AF, Miyasaka et $a l^{24}$ observed the highest prevalence of thromboembolism $(28 \%)$ in those with grade $1 \mathrm{MR}$, whereas those with grade $\geq 2(8 \%, \mathrm{p}=0.006)$ and with none had the lowest event rates $(11 \%, \mathrm{p}=0.007)$. Platelet activation due to MR may enhance the formation of plateletfibrin thrombus on the mitral leaflet, thereby increasing embolisation. $^{26}$

In contrast to some of the reports mentioned above, we could not confirm a reduced thromboembolism rate in our patients with MR compared with no SVD.

\section{Safety outcomes}

Current literature lacks detailed analyses of bleeding in valve disease patients taking oral anticoagulants to prevent stroke. In our study, patients with AS had the highest risk of major bleeding in comparison with patients with no SVD $(p<0.05)$ but not significantly higher compared with patients with MR or AR. The latter patients also had a significantly higher rate of major bleeding than patients with no SVD $(\mathrm{p}<0.01)$. 
Figure 3 Treatment comparisons between patients allocated to rivaroxaban and warfarin. Treatment comparisons between patients allocated to rivaroxaban and warfarin for safety endpoints among SVD subtypes and for patients with no SVD. Events per 100 patient-years, unadjusted. $\mathrm{p}$ Values for any difference among groups are based on Cox proportional hazards models. Patients in each treatment are shown separately. For results of detailed statistical analyses, see online supplementary table S1. NMCR, non-major clinically relevant; pt-years, patient years; SVD, significant valve disease; MR or AR, mitral or aortic regurgitation.

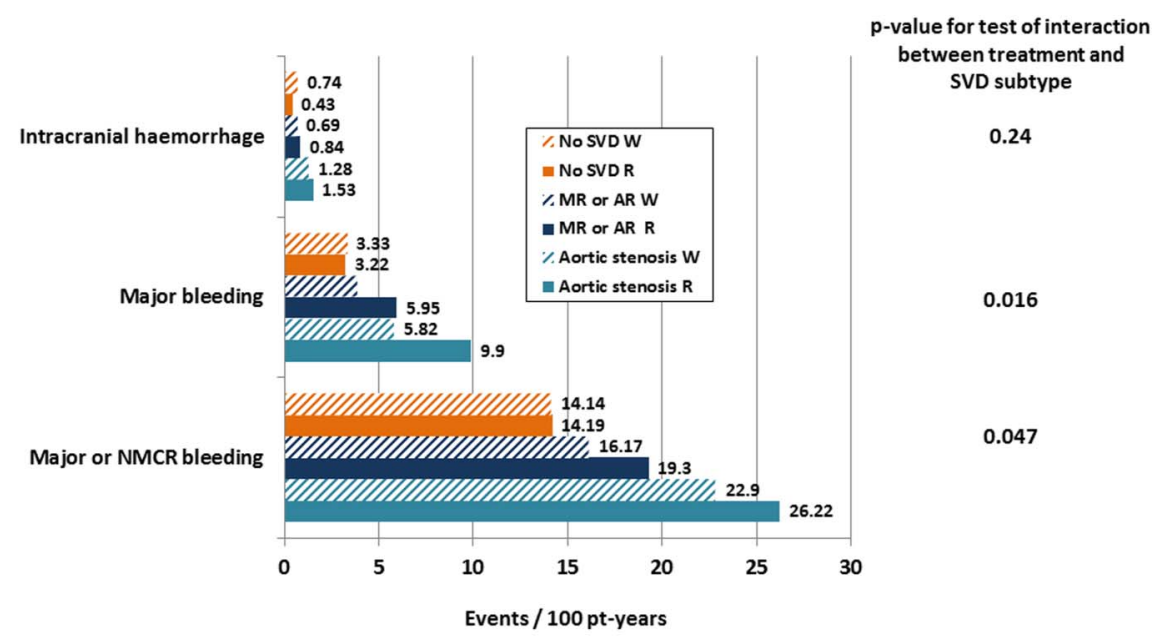

Cox proportional hazards models
Our major bleeding rate of 3.27 events per 100 patient-years in patients with no SVD (table 3 ) is close to real-world data from a pharmacovigilance study of rivaroxaban with non-valvular AF that excluded patients with any type of valve disease. In this study of 27467 patients, the incidence of major bleeding was 2.86 per 100 person-years (95\% CI 2.61 to 3.13$).{ }^{27} \mathrm{CHADS}_{2}$ scores were 3.0 for those with major bleeding and 2.2 for those without; these scores are lower than those in our population.

\section{Mechanisms of thromboembolism and of bleeding in valvular disease}

Potential mechanisms for systemic embolism in AS include increased mean platelet volume, ${ }^{28}$ increased platelet production indices in aortic valve sclerosis patients ${ }^{29}$ and turbulent flow with subsequent activation of coagulation and platelets. ${ }^{30}$ Minimally calcified aortic valves have proven to exhibit anti-aggregatory effects, whereas AS does not demonstrate these same effects. ${ }^{31}$ In addition to a possible prothrombotic effect, other phenomena observed in patients with AS demonstrate evidence of impaired haemostasis with a decrease in von Willebrand factor. ${ }^{32}$ Concomitant AF further complicates the picture since AF is consistently related to higher levels of von Willebrand factor than in AF's absence. ${ }^{33-36}$ Consequently, there may be opposing disturbances in the coagulation system due to the AS/AF combination.

We observed an increased bleeding risk in patients with AS, which was more pronounced on rivaroxaban than warfarin; if this is not a chance finding, then it is of clinical importance and needs further corroboration, yet our data do not explain the mechanisms behind the potentially higher bleeding rate in patients with AS.

Bleeding in patients with AS has been related to gastrointestinal angiodysplasia (ie, Heyde's syndrome), ${ }^{37-40}$ which is associated with acquired type $2 \mathrm{~A}$ von Willebrand syndrome (characterised by the loss of the largest multimers of von Willebrand factor). ${ }^{4142}$ Shear stress ex vivo enhances the proteolysis of von Willebrand factor in normal plasma. ${ }^{42}$ Vincentelli et $a l^{32}$ found von Willebrand factor abnormalities to be directly related to the stenosis severity; furthermore, they could be reversed by aortic valve replacement if there was no implanted prosthesis mismatch. ${ }^{32} 43-46$

\section{Effect of type of anticoagulation}

For efficacy outcomes, there were no significant interactions since the interaction tests between treatment and SVD group were non-significant; therefore, the risk relationship between rivaroxaban and warfarin was consistent across SVD subgroups. HRs from the full ROCKET AF cohort ${ }^{2}$ are the best estimate of treatment effects.

Interaction tests for between-treatment bleeding suggested a significant difference when comparing the three subgroups. In patients without SVD, rivaroxaban and warfarin had a neutral relationship (HR close to 1.0), whereas those with MR or AR had an elevated risk of any bleeding (except for intracranial haemorrhage) with rivaroxaban compared with warfarin. Patients with AS also had higher HR for rivaroxaban versus warfarin, which was especially pronounced for major bleeding (this difference did not reach statistical significance; CIs were below and above 1.0). There was a tendency towards higher rates of gastrointestinal bleeding in patients with AS and MR or AR. There were too few intracranial haemorrhages to draw any conclusions.

Our study had several limitations. First, although we examined a large trial population, the proportion of patients with moderate or intermediate AS severity was relatively small. Second, ${ }^{3}$ the protocol did not include a precise quantification of valve disease, so the two SVD subgroups do not represent isolated disease types (eg, patients in the AS group also had regurgitant lesions). Third, our data do not allow any balance estimate between AS severity and other lesions (if present). Finally, the relatively low number of cases, especially in those with AS, reduces the chance of finding true differences. Therefore, conclusions regarding treatment effects should be drawn cautiously. More studies are needed to better understand the thromboembolic event-related clinical characteristics and outcomes in patients with valvular disease (after exclusion of mitral stenosis or artificial valves).

\section{CONCLUSIONS}

Our retrospective exploratory analysis of ROCKET AF found that among patients with AF treated with oral anticoagulation (either rivaroxaban or warfarin), AS stood out from other groups with regard to efficacy endpoints (eg, stroke, systemic embolism and vascular death); in patients with other valve disease (MR or $\mathrm{AR}$ ) and non-valve disease (no SVD), these endpoints did not differ. Major bleeding rates increased from no SVD to MR or AR to AS. Oral anticoagulation type did not cause a difference in efficacy outcomes, but bleeding occurred more frequently in patients with AS and other types of valve disease randomised to 
rivaroxaban rather than warfarin (although the difference missed significance). There was no difference in the no SVD group between treatment allocation. We believe our study is the first to suggest that in patients with AF who mainly have AS treated with oral anticoagulation, the efficacy and safety outcomes may be different from those with MR or AR (predominantly MR) or no SVD. This notion may be intuitive, but has not been demonstrated in a patient population selected for AF presence.

\section{Key messages}

What is already known on this subject?

Current literature is lacking detailed analyses of various types of valve disease (after exclusion of patients with mitral stenosis or artificial prostheses) in the presence of atrial fibrillation with regard to systemic embolism and bleeding when a patient is on warfarin or rivaroxaban for stroke prevention.

\section{What might this study add?}

In this first detailed analysis of subgroups with valve disease, the composite of stroke, systemic embolism or vascular death was about twofold higher in aortic stenosis (AS) than in mitral or aortic regurgitation or no valve disease. Major bleeding occurred more frequently in AS than in the other two groups. For efficacy endpoints comparing rivaroxaban and warfarin, there were no significant treatment interactions but for bleeding outcomes, the interaction tests were significant.

\section{How might this impact on clinical practice?}

We found that patients with atrial fibrillation and AS who are being treated with oral anticoagulation have efficacy and safety outcomes that are distinctly different from patients with other types or without any significant valve disease; this finding should encourage clinicians to carefully consider the risks of thromboembolic complications versus bleeding especially in patients with AS.

\section{Author affiliations}

${ }^{1}$ Division of Electrophysiology, Department of Cardiovascular Medicine, University Hospital Münster, Münster, Germany

${ }^{2}$ Division of Adult Congenital and Valvular Heart Disease, Department of Cardiovascular Medicine, University Hospital Münster, Münster, Germany ${ }^{3}$ Bayer Healthcare Pharmaceuticals, L.P., Whippany, New Jersey, USA

${ }^{4}$ Duke Clinical Research Institute, Duke University Medical Center, Durham, North Carolina, USA

${ }^{5}$ The Cardiovascular Institute, Mount Sinai Medical Center, New York, USA ${ }^{6}$ Clinical Epidemiology Unit, Division of General Internal Medicine, Massachusetts General Hospital, and Harvard Medical School, Boston, Massachusetts, USA ${ }^{7}$ School of Medicine and Pharmacology, The University of Western Australia, Perth, Australia

${ }^{8}$ Ruprecht-Karls-University, Heidelberg, Germany

${ }^{9}$ Division of Cardiovascular Health and Disease, Department of Medicine, University of Cincinnati College of Medicine, Cincinnati, Ohio, USA

${ }^{10} J o h n s o n$ \& Johnson Pharmaceutical Research \& Development, Raritan, New Jersey, USA

${ }^{11}$ Department of Medicine, Stanford University, Stanford, California, USA

${ }^{12}$ Duke Translational Medicine Institute, Duke University Medical Center, Durham, North Carolina, USA

${ }^{13}$ University of Edinburgh, and Royal Infirmary of Edinburgh, Edinburgh, UK

Contributors GB had full access to all of the data in the study and takes responsibility for the integrity of the data and the accuracy of the data analysis. GB contributed to the conception and design of the study, the data analysis, the data interpretation, the manuscript drafting and the critical revision of the manuscript. $H B, S D B, J P P, Y L, J L H, D E S, G J H, W H, R C B, C C N, K W M, R M C$ and KAAF contributed to the data analysis, the data interpretation, the manuscript drafting and the critical revision of the manuscript. ASH was the responsible biostatistician who contributed to the data analysis, the data interpretation, the manuscript drafting and the critical revision of the manuscript. MRP contributed to the conception and design of the study, the supervision, data acquisition, analysis and interpretation, the manuscript drafting and the critical revision of the manuscript.

Funding The ROCKET AF trial was supported by research grants from Janssen Research \& Development (Raritan, New Jersey, USA) and Bayer HealthCare AG (Leverkusen, Germany).

Competing interests GB: consultant to Bayer Health Care, J\&J, Boehringer Ingelheim, Sanofi-Aventis, MSD, 3M. SDB: employed by Bayer HealthCare Pharmaceuticals. JPP: consulting fees/honoraria for Bayer, Johnson \& Johnson, Medtronic and Spectranetics; research grants from ARCA Biopharma, Boston Scientific, Gilead, Johnson \& Johnson and ResMed. YL: consulting fees from Johnson \& Johnson. MRP: consulting fees from Bayer Healthcare, Ortho McNeil Jansen, Medscape-theheart.org, Ikaria; research grants from Johnson and Johnson, Maquet, Astra Zeneca, National Heart Lung and Blood Institute, Genzyme. JL: consulting fees from Boehringer Ingelheim, Sanofi-Aventis, Biotronik, Inc, Ortho-McNeil-Janssen Pharmaceuticals, Inc, Johnson \& Johnson, Medtronik, Inc, AstraZeneca, Daiich Sankyo Pharma, Pfizer, Inc, Bristol Meyers Squibb, Bayer HealthCare AG. DES: consulting fees from Boehringer Ingelheim, Bristol-Myers Squibb, Johnson and Johnson, Inc, Pfizer, Daiichi Sankyo, Medtronic, Inc, Bayer Healthcare. GJH: consulting fees from Duke Clinical Research Institute, Bayer Healthcare and Medscape-the heart.org. WH: consulting fees from Sygnis Pharma Germany, Photothera USA, Boehringer Ingelheim, Codman USA and Bayer; research grants from Boehringer Ingelheim. RCB: consulting fees from Bristol-Myers Squibb, Sanofi-Aventis, Boehringer Ingelheim; research grants from BMS, Bayer Pharmaceuticals, Johnson and Johnson, Regado Biosciences, AstraZeneca. CCN: employed by Johnson \& Johnson Pharmaceutical Research and Development. KWM: full disclosures prior to 1 August 2013 available at http://www.dcri.org. Disclosures after 1 August 2013 available at http://med.stanford.edu/profiles/kenneth mahaffey. KAAF: consulting fees from Boehringer Ingelheim, Sanofi-Aventis, Astra Zeneca, Johnson \& Johnson/Bayer; research grants from Eli Lilly. RMC: consulting fees from KOWA, Eli Lilly, GlaxoSmithKline, WebMD, Bristol Myers Squibb, Nitrox LLC, Bayer, Orexigen Therapeutics, Sanofi-Aventis, Medtronic, Boehringer Ingelheim, Gilead: research grants from BMS, Roche, Merck, Merck, Novartis, Scios/Johnson and Johnson, Amilyn, Bristol Myers Squibb, Bayer.

Ethics approval The IRB application for the original research protocol submitted by the Rocket AF DCRI Coordinating Center to the Duke Institutional Review Board had the protocol number Pro00017525. This secondary analysis was approved under protocol number Pro00032515 by the same IRB.

Provenance and peer review Not commissioned; externally peer reviewed.

Data sharing statement This is a substudy of ROCKET AF. In a similar way, several substudies have been published since publication of the main manuscript (reference ${ }^{2}$ ).

Open Access This is an Open Access article distributed in accordance with the Creative Commons Attribution Non Commercial (CC BY-NC 4.0) license, which permits others to distribute, remix, adapt, build upon this work non-commercially, and license their derivative works on different terms, provided the original work is properly cited and the use is non-commercial. See: http://creativecommons.org/ licenses/by-nc/4.0/

\section{REFERENCES}

1 ROCKET AF Study Investigators. Rivaroxaban-once daily, oral, direct factor Xa inhibition compared with vitamin $\mathrm{K}$ antagonism for prevention of stroke and Embolism Trial in Atrial Fibrillation: rationale and design of the ROCKET AF study. Am Heart J 2010;159:340-7.e1.

2 Patel MR, Mahaffey KW, Garg J, et al. Rivaroxaban versus warfarin in nonvalvular atrial fibrillation. N Engl J Med 2011:365:883-91.

3 Breithardt G, Baumgartner H, Berkowitz SD, et al. Clinical characteristics and outcomes with rivaroxaban vs. warfarin in patients with non-valvular atrial fibrillation but underlying native mitral and aortic valve disease participating in the ROCKET AF trial. Eur Heart J 2014:35:3377-85.

4 Hohnloser SH, Lopes RD. Atrial fibrillation, valvular heart disease, and use of target-specific oral anticoagulants for stroke prevention. Eur Heart J 2014;35:3323-5.

5 Avezum A, Lopes RD, Schulte PJ, et al. Apixaban in comparison with warfarin in patients with atrial fibrillation and valvular heart disease: findings from the apixaban for reduction in stroke and other thromboembolic events in atrial fibrillation (ARISTOTLE) trial. Circulation 2015;132:624-32.

6 Ezekowitz MD, Parise $H$, Nagarakanti $R$, et al. Comparison of dabigatran and warfarin in patients with atrial fibrillation and valvular heart disease: the RE-LY trial [Abstract]. J Am Coll Cardiol 2014;63:A325.

7 Breithardt G, Baumgartner H, Berkowitz SD, et al. Patients with native aortic stenosis represent a high-risk subgroup in nonvalvular atrial fibrillation-results from ROCKET AF[Abstract 5835]. Eur Heart J 2014;35(Abstract Supplement): 1033 . 
8 Otto CM, Lind BK, Kitzman DW, et al. Association of aortic-valve sclerosis with cardiovascular mortality and morbidity in the elderly. N Eng/ J Med 1999;341:142-7.

9 Greve AM, Dalsgaard M, Bang CN, et al. Stroke in patients with aortic stenosis: the Simvastatin and Ezetimibe in Aortic Stenosis study. Stroke 2014;45:1939-46.

10 Baskett RJ, Exner DV, Hirsch GM, et al. Mitral insufficiency and morbidity and mortality in left ventricular dysfunction. Can J Cardiol 2007;23:797-800. doi:10. 1016/S0828-282X(07)70830-9

11 Koelling TM, Aaronson KD, Cody RJ, et al. Prognostic significance of mitral regurgitation and tricuspid regurgitation in patients with left ventricular systolic dysfunction. Am Heart J 2002;144:524-9.

12 Robbins JD, Maniar PB, Cotts W, et al. Prevalence and severity of mitral regurgitation in chronic systolic heart failure. Am J Cardiol 2003;91:360-2.

13 Shimada S. A 13-year follow-up study of rheumatic valvular diseases. Jpn Circ J 1986;50:1304-8

14 Bonow RO, Carabello B, de Leon AC Jr, et al. ACC/AHA guidelines for the management of patients with valvular heart disease: executive summary: a report of the American College of Cardiology/American Heart Association Task Force on Practice Guidelines (Committee on Management of Patients With Valvular Heart Disease). Circulation 1998;98:1949-84.

15 Salem DN, Levine HJ, Pauker SG, et al. Antithrombotic therapy in valvular heart disease: antithrombotic therapy and prevention of thrombosis, 9th ed: American College of Chest Physicians Evidence-Based Clinical Practice Guidelines. Chest 1998;114:e590S-601S

16 [No authors listed]. Cardiogenic brain embolism. Cerebral Embolism Task Force. Arch Neurol 1986;43:71-84.

17 Vahanian A, Alfieri 0 , Andreotti F, et al. Guidelines on the management of valvular heart disease (version 2012). Eur Heart J 2012;33:2451-96.

18 Petty GW, Khandheria BK, Whisnant JP, et al. Predictors of cerebrovascular events and death among patients with valvular heart disease: a population-based study. Stroke 2000;31:2628-35.

19 Davidsen B, Egeblad H, Pietersen A. Thromboembolism in patients with advanced mitral valve prolapse. J Intern Med 1989;226:433-6.

20 Davison G, Greenland P. Predictors of left atrial thrombus in mitral valve disease. J Gen Intern Med 1991;6:108-12.

21 Movsowitz C, Movsowitz HD, Jacobs LE, et al. Significant mitral regurgitation is protective against left atrial spontaneous echo contrast and thrombus as assessed by transesophageal echocardiography. J Am Soc Echocardiogr 1993:6:107-14.

22 Blackshear JL, Pearce LA, Asinger RW, et al. Mitral regurgitation associated with reduced thromboembolic events in high-risk patients with nonrheumatic atrial fibrillation. Stroke Prevention in Atrial Fibrillation Investigators. Am J Cardiol 1993;72:840-3.

23 Karatasakis GT, Gotsis AC, Cokkinos DV. Influence of mitral regurgitation on left atrial thrombus and spontaneous echocardiographic contrast in patients with rheumatic mitral valve disease. Am J Cardiol 1995;76:279-81.

24 Miyasaka Y, Tsuji H, Tokunaga S, et al. Mild mitral regurgitation was associated with increased prevalence of thromboembolic events in patients with nonrheumatic atrial fibrillation. Int J Cardiol 2000;72:229-33.

25 Nair CK, Aronow WS, Shen $\mathrm{X}$, et al. Effect of mitral regurgitation on cerebrovascular accidents in patients with atrial fibrillation and left atrial thrombus. Clin Cardiol 2009;32:E7-E10
26 Tse HF, Lau CP, Cheng G. Relation between mitral regurgitation and platelet activation. J Am Coll Cardiol 1997;30:1813-18.

27 Tamayo S, Peacock WF, Patel M, et al. Characterizing major bleeding in patients with nonvalvular atrial fibrillation: a pharmacovigilance study of 27,467 patients taking rivaroxaban. Clin Cardiol 2015;38:63-8.

28 Varol E, Arslan A, Yucel H, et al. Increased mean platelet volume in patients with aortic stenosis. Clin Appl Thromb Hemost 2011;17:E17-20.

29 Sucu M, Davutoglu V, Sari l, et al. Relationship between platelet indices and aortic valve sclerosis. Clin Appl Thromb Hemost 2010;16:563-7.

30 Dimitrow PP, Hlawaty M, Undas $A$, et al. Effect of aortic valve stenosis on haemostasis is independent from vascular atherosclerotic burden. Atherosclerosis 2009;204:e103-8.

31 Chirkov YY, Mishra K, Chandy S, et al. Loss of anti-aggregatory effects of aortic valve tissue in patients with aortic stenosis. $J$ Heart Valve Dis 2006;15:28-33.

32 Vincentelli A, Susen S, Le Tourneau T, et al. Acquired von Willebrand syndrome in aortic stenosis. N Engl J Med 2003;349:343-9.

33 Lip GY, Lowe GD, Rumley A, et al. Increased markers of thrombogenesis in chronic atrial fibrillation: effects of warfarin treatment. Br Heart J 1995;73:527-33. doi:10. 1136/hrt.73.6.527

34 Freestone B, Gustafsson F, Chong AY, et al. Influence of atrial fibrillation on plasma von Willebrand factor, soluble E-selectin, and N-terminal pro B-type natriuretic peptide levels in systolic heart failure. Chest 2008;133:1203-8.

35 Gustafsson C, Blombäck M, Britton M, et al. Coagulation factors and the increased risk of stroke in non-valvular atrial fibrillation. Stroke 1990;21:47-51.

36 Li-Saw-Hee FL, Blann AD, Lip GY. Effects of fixed low-dose warfarin, aspirin-warfarin combination therapy, and dose-adjusted warfarin on thrombogenesis in chronic atrial fibrillation. Stroke 2000;31:828-33.

37 Heyde EC. Gastrointestinal bleeding in aortic stenosis. N Engl J Med 1958;259:196.

38 Greenstein RJ, McElhinney Al, Reuben D, et al. Colonic vascular ectasias and aortic stenosis: coincidence or causal relationship? Am J Surg 1986;151:347-51.

39 King RM, Pluth JR, Giuliani ER. The association of unexplained gastrointestinal bleeding with calcific aortic stenosis. Ann Thorac Surg 1987;44:514-16.

40 Pate GE, Mulligan A. An epidemiological study of Heyde's syndrome: an association between aortic stenosis and gastrointestinal bleeding. J Heart Valve Dis 2004;13:713-6

41 Warkentin TE, Moore JC, Morgan DG. Aortic stenosis and bleeding gastrointestinal angiodysplasia: is acquired von Willebrand's disease the link? Lancet 1992;340:35-7.

42 Tsai HM, Sussman II, Nagel RL. Shear stress enhances the proteolysis of von Willebrand factor in normal plasma. Blood 1994;83:2171-9.

43 Anderson RP, McGrath K, Street A. Reversal of aortic stenosis, bleeding gastrointestinal angiodysplasia, and von Willebrand syndrome by aortic valve replacement. Lancet 1996;347:689-90.

44 Pareti FI, Lattuada A, Bressi C, et al. Proteolysis of von Willebrand factor and shear stress-induced platelet aggregation in patients with aortic valve stenosis. Circulation 2000;102:1290-5.

45 Thompson JL III, Schaff HV, Dearani JA, et al. Risk of recurrent gastrointestinal bleeding after aortic valve replacement in patients with Heyde syndrome. J Thoracic Cardiovasc Surg 2012;144:112-16.

46 Loscalzo J. Basic implications of clinical observations: from clinical observation to mechanism-Heyde's Syndrome. N Engl J Med 2012;367:1954-6. 